\title{
Searches for BSM Higgs bosons in fermionic decays in ATLAS
}

Adam Bailey* on behalf of the ATLAS collaboration

Instituto de Fisica Corpuscular (IFIC), Centro Mixto Universidad de Valencia - CSIC, Spain

E-mail: adam.bailey@cern.ch

Beyond the Standard Model theories such as the MSSM and other two-Higgs-doublet-models predict an extended Higgs sector. In some scenarios the additional Higgs bosons can have significant branching ratios to fermions. The ATLAS detector at the LHC has set stringent limits on beyond the Standard Model Higgs bosons from $36.1 \mathrm{fb}^{-1}$ of proton-proton collision data collected in Run 2. This report summarises some of the searches for heavy neutral beyond the Standard Model Higgs bosons in $\tau$ and top final states.

Prospects for Charged Higgs Discovery at Colliders - CHARGED2018

25-28 September 2018

Uppsala, Sweden

${ }^{*}$ Speaker. 


\section{Introduction}

Despite the success of the Standard Model in describing experimental results, there are several reasons as to why it may be incomplete. For example, no particle in the Standard Model has suitable properties to be the dark matter observed in the universe, there is the hierarchy problem associated with the Higgs mass, and currently no method to incorporate gravity. The three fundamental forces included also do not converge at high energy. Beyond the Standard Model (BSM) theories seek to address these shortcomings. Many BSM theories predict an extended Higgs sector, with multiple Higgs bosons in addition to the one at $125 \mathrm{GeV}[1,2]$. This report addresses the search for heavy neutral BSM Higgs bosons in fermionic decay channels, with the ATLAS detector at the LHC.

In general the searches are for two Higgs doublet models (2HDM) where an additional Higgs doublet leads to a total of five Higgs bosons [3]. Three of these are neutral (two of those CP even, one CP odd), and two charged. One of the neutral CP even Higgs must be consistent with the observation of the Higgs at $125 \mathrm{GeV}$. One such $2 \mathrm{HDM}$ is the minimal supersymmetric model (MSSM) [4]. At tree level the MSSM can be described by the mass of the CP-odd neutral Higgs $\left(m_{A}\right)$ and the ratio of the vacuum expectation values of the two Higgs doublets $(\tan \beta)$.

Three benchmark MSSM models studied in these results: hMSSM [5, 6], $m_{h}^{\bmod +}$, and $m_{h}^{\bmod -}$ [7]. In hMSSM the mass of the observed Higgs boson is used to predict the remaining masses and couplings in the model. The $m_{h}^{\bmod }$ models originate from the $m_{h}^{\max }$ model, which maximised $m_{h}$ for a given $m_{A}$ and $\tan \beta$. The mixing in the top sector in $m_{h}^{\max }$ was modified to enable $m_{h}$ to be consistent with $125 \mathrm{GeV}$, and leads to the $m_{h}^{\bmod +}$ and $m_{h}^{\bmod -}$ models. In addition, modelindependent limits are also presented.

The most relevant production modes for BSM Higgs bosons in proton-proton collisions at the LHC are gluon-gluon fusion $(g g \mathrm{~F})$ and $b$-associated $(b b H)$ production, shown in Fig. 1 . When $\tan \beta$ is low, the $g g \mathrm{~F}$ production is the dominant process. As $\tan \beta$ is increased, the $b b H$ channel dominates. The branching fractions to fermions also depend on $\tan \beta$. For $m_{A}$ above approximately $350 \mathrm{GeV}$ and low $\tan \beta$, the dominant decay is into $t \bar{t}$. At high $\tan \beta$ the Higgs primarily decays into $b \bar{b}$ or $\tau \tau$. Although $b \bar{b}$ has large branching fractions it also has large multijet backgrounds. Therefore, ATLAS searches in fermionic channels so far have focused on top and $\tau \tau$ final states.

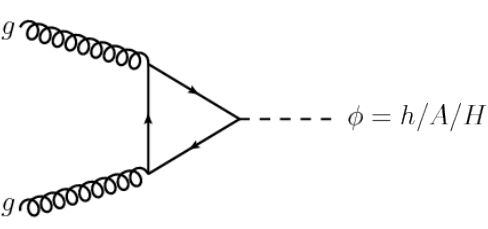

(a)

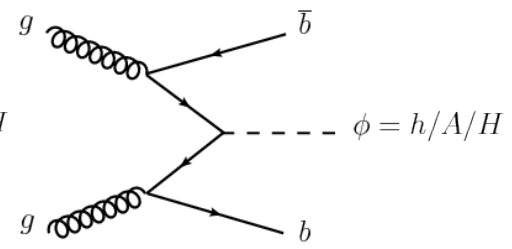

(b)

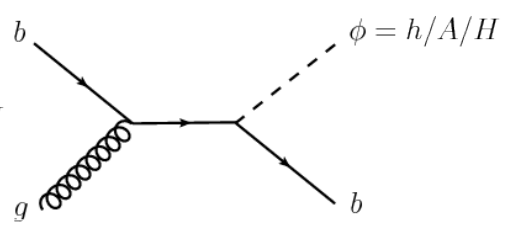

(c)

Figure 1: Lowest order Feynman diagrams for neutral BSM Higgs production. (a) Gluon-gluon fusion. (b) $b$-associated production. (c) $b$-associated production in the 5 -flavour scheme.

\section{H/A to $\tau \tau$ search}

The latest BSM Higgs to $\tau \tau$ search from ATLAS [8] used $36 \mathrm{fb}^{-1}$ of proton-proton collision data at a centre of mass energy of $13 \mathrm{TeV}$, collected during 2015 and 2016. This channel is par- 
ticularly sensitive at high $\tan \beta$ where both the branching to $\tau$ is enhanced, and the production in association with $b$-quarks. The $\tau$ leptons decay either leptonically $\left(\tau_{\text {lep }}, 35 \%\right)$ or hadronically ( $\tau_{\text {had }}$, $65 \%$ ). A hadronic $\tau$ decay produces charged and neutral hadrons (primarily pions), and a neutrino. The analysis is split into a fully hadronic channel ( $\left.\tau_{\text {had }} \tau_{\text {had }}\right)$ and semi-leptonic channel $\left(\tau_{\text {lep }} \tau_{\text {had }}\right)$. Both the $g g \mathrm{~F}$ and $b b H$ production are considered, which motivates a further split into $b$-tag and $b$-veto categories.

The signal topology is a pair of back-to-back, oppositely charged $\tau$, with some missing transverse momentum, $\mathbf{E}_{\mathrm{T}}^{\mathrm{miss}}$ (from neutrinos in the $\tau$ decays). Events are triggered using a single lepton trigger (with thresholds between $20-140 \mathrm{GeV}$ ) in the $\tau_{\text {lep }} \tau_{\text {had }}$ channel, or a hadronic $\tau$ trigger (threshold of 80,125 , or $160 \mathrm{GeV}$, depending on the data taking period) in the $\tau_{\text {had }} \tau_{\text {had }}$ channel. Hadronic $\tau$ decays are identified using multivariate analysis to distinguish the objects from jets [9]. In the fully hadronic channel two BDT requirements are used: a medium ID on the lead $\tau$, which achieves a 75\% (60\%) efficiency with a factor 50 (100) background rejection for 1 (3) track $\tau$, and a loose ID on the sub-leading $\tau$, which achieves a $85 \%$ (75\%) efficiency with a factor 30 (30) background rejection for 1 (3) track $\tau$. The semi-leptonic channel also requires a medium ID on the $\tau_{\text {had }}$, and has varying lepton isolation requirements on the $\tau_{\text {lep }}$ depending on which trigger was used. A full description of the selection for both channels and the requirements for the $b$-tag or $b$-veto category can be found in Ref. [8].

A main background in both channels comes from multijet events, where jets fake $\tau_{\text {had }}$ or leptons. In the $\tau_{\text {lep }} \tau_{\text {had }}$ channel there is also the $W+$ jets background with a real lepton and a fake $\tau_{\text {had }}$ from a jet. These backgrounds are estimated using a data-driven fake factor method. For the $\tau_{\text {lep }} \tau_{\text {had }}$ channel the other backgrounds are $Z / \gamma^{*} \rightarrow \tau \tau$ or $\rightarrow t \bar{t}$ (real $\tau$ in final state), $Z / \gamma^{*} \rightarrow \ell \ell$ (real lepton with fake $\tau_{\text {had }}$ ), diboson, and single top. These are estimated using simulation. For $\tau_{\text {had }} \tau_{\text {had }}$ other significant backgrounds are from $Z / \gamma^{*} \rightarrow \tau \tau$ (real $\tau$ ), $W \rightarrow \tau \nu+$ jets (real $\tau$ with jet fake), $t \bar{t}$ (mix of real $\tau$ and lepton/jet fakes), $W \rightarrow \ell v+$ jets, $Z / \gamma^{*} \rightarrow \ell \ell+$ jets, and diboson. These are also estimated from simulation, but with a data driven fake rate measurement to estimate the probability of a jet faking a $\tau$.

The statistical test employed is a likelihood function, constructed as the product of Poisson probability terms. The parameter of interest is $\mu$, the ratio of the observed to the predicted value of the cross section times branching fraction, evaluated for a particular model under test over a range of points in the parameter space. The fit of the MSSM model to data is performed separately for the $b$-tag and $b$-veto categories, in order to enhance the sensitivity to the $b b H$ and $g g F$ production mode respectively. Systematic uncertainties on the signal and background predictions are parameterised as nuisance parameters that are constrained using Gaussian probability density functions. The observed event yields are compatible with that expected from the Standard Model backgrounds and the post-fit distributions are shown in Fig. 2. The variable used to present the results is $m_{\mathrm{T}}^{\text {tot }}$, which is defined as:

$$
m_{\mathrm{T}}^{\text {tot }}\left(\tau_{1}, \tau_{2}, \mathbf{E}_{\mathrm{T}}^{\text {miss }}\right)=\sqrt{m_{\mathrm{T}}^{2}\left(\tau_{1}, \tau_{2}\right)+m_{\mathrm{T}}^{2}\left(\tau_{1}, \mathbf{E}_{\mathrm{T}}^{\text {miss }}\right)+m_{\mathrm{T}}^{2}\left(\tau_{2}, \mathbf{E}_{\mathrm{T}}^{\text {miss }}\right)},
$$

where $\tau_{1}$ and $\tau_{2}$ are the leading and subleading $\tau$ in transverse momentum respectively.

From this limits are set on the branching fraction times cross section to $\tau \tau$ for the $g g \mathrm{~F}$ and $b b H$ production of a BSM Higgs, as shown in Fig. 3. Furthermore, limits on particular models are set in 


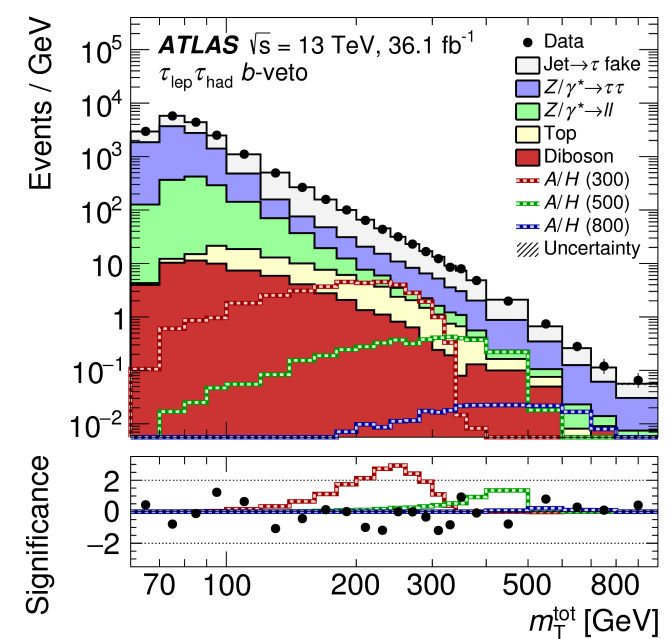

(a)

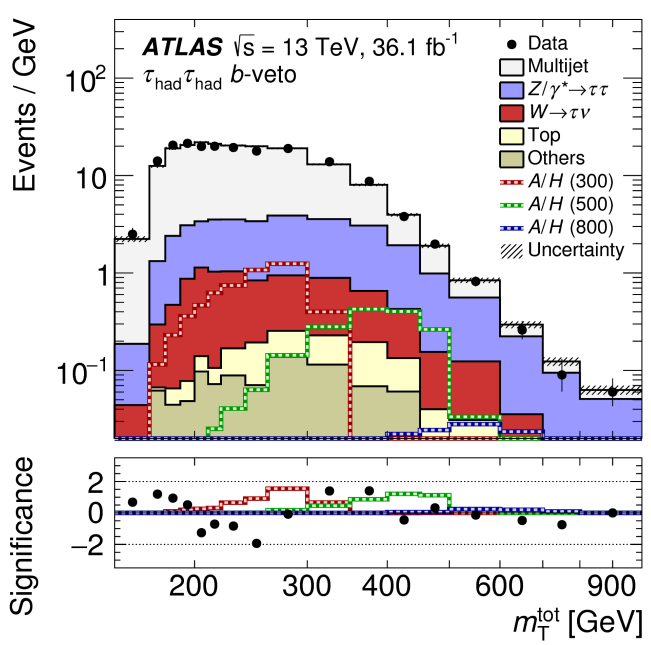

(c)

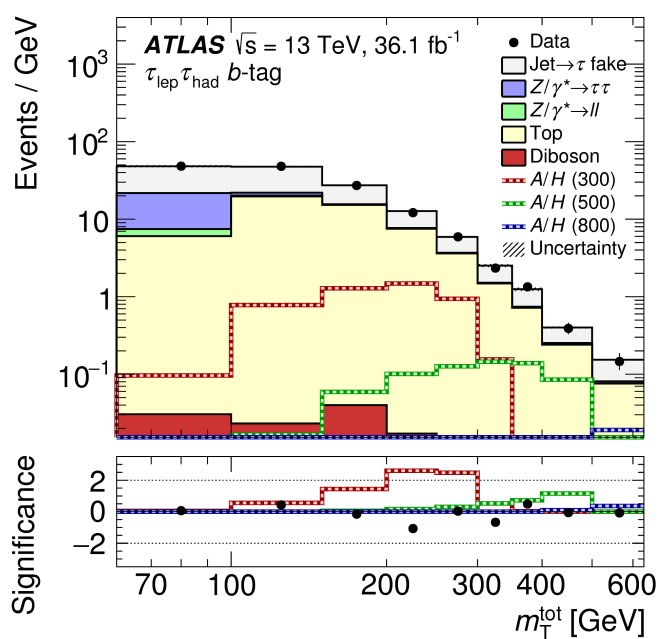

(b)
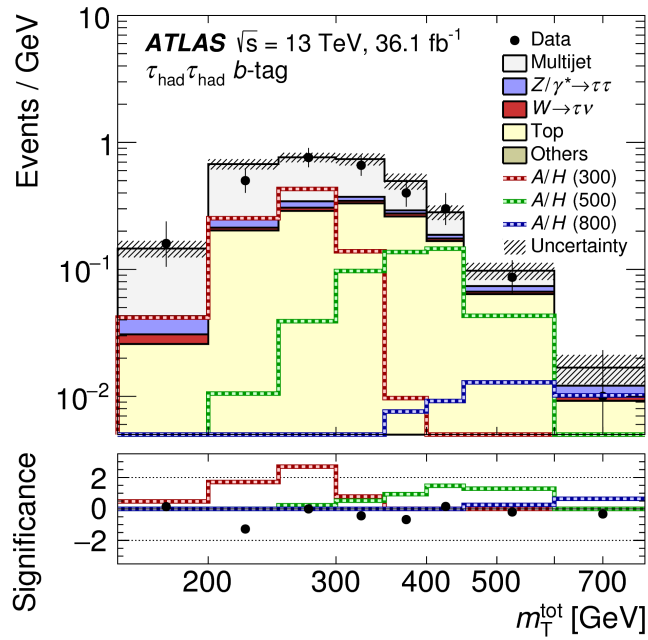

(d)

Figure 2: Postfit $m_{\mathrm{T}}^{\text {tot }}$ distributions from the BSM neutral Higgs to $\tau \tau$ search. The predicted signals for $\mathrm{A}$ and $\mathrm{H}$ bosons with masses of 300,400 and $800 \mathrm{GeV}$ and $\tan \beta=10$ are also included. (a) $\tau_{\text {lep }} \tau_{\text {had }} b$-veto category. (b) $\tau_{\text {lep }} \tau_{\text {had }} b$-tag category. (c) $\tau_{\text {had }} \tau_{\text {had }} b$-veto category. (d) $\tau_{\text {had }} \tau_{\text {had }}$ $b$-tag category [8].

the $\tan \beta$ against $m_{A}$ plane. Fig. 4 shows the limits obtained for the $m_{h}^{\bmod +}$ and hMSSM scenarios. 


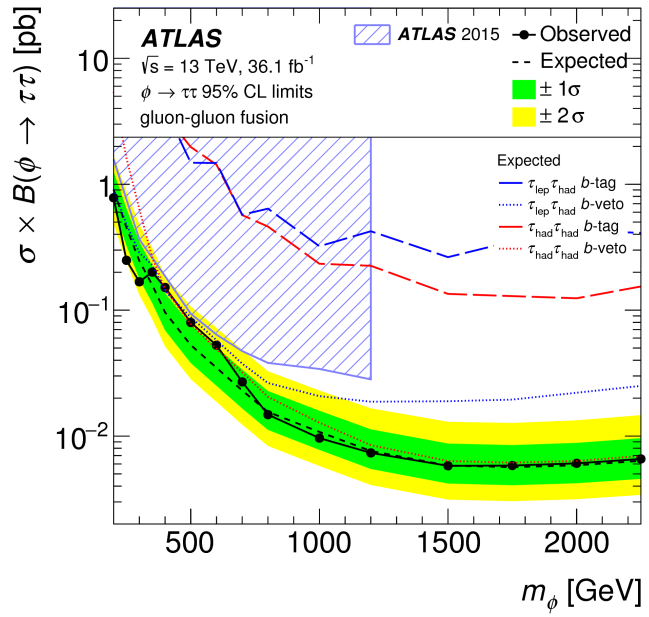

(a)

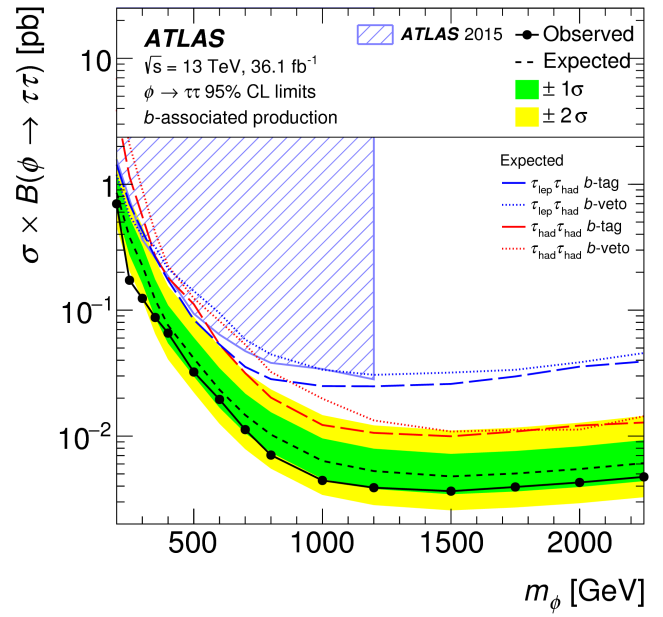

(b)

Figure 3: Observed and expected 95\% CL upper limits on the production cross section times branching fraction for a scalar boson produced via (a) gluon-gluon fusion and (b) $b$-associated production. The contribution to the expected limits from the individual channels is also shown. The blue shaded area shows the previous ATLAS limit from 2015 data [8].

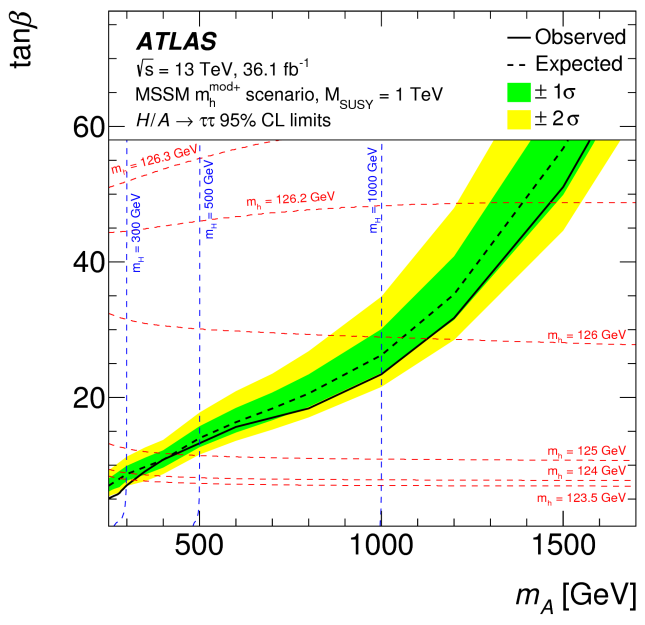

(a)

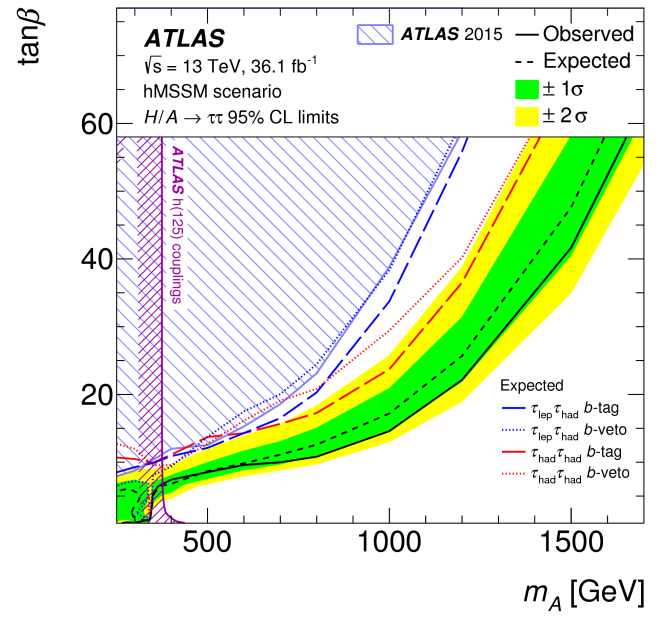

(b)

Figure 4: Observed and expected 95\% CL upper limits in the $\tan \beta$ against $m_{A}$ plane for the MSSM. (a) $m_{h}^{\bmod +}$ scenario. The dashed red and blue lines are lines of constant $\mathrm{mh}$ and $\mathrm{mH}$ respectively. (b) hMSSM scenario. The blue shaded area shows the ATLAS limit from 2015 [8].

\section{Searches with top final states}

Searches with top final states are more sensitive for models where $\tan \beta$ is low, so are complementary to $\tau$ final states. ATLAS carried out a direct search for the BSM Higgs to $t \bar{t}$ decay with $20.3 \mathrm{fb}^{-1}$ of data at $8 \mathrm{TeV}$ [10]. It is affected by interference between the signal and standard 
model $t \bar{t}$ backgrounds. The interference gives a characteristic "peak and dip" structure, which is a unique signature. However, care must be taken to ensure this interference is properly modelled and accounted for. An example of the expected signal shape accounting for the interference is shown in Fig 5.

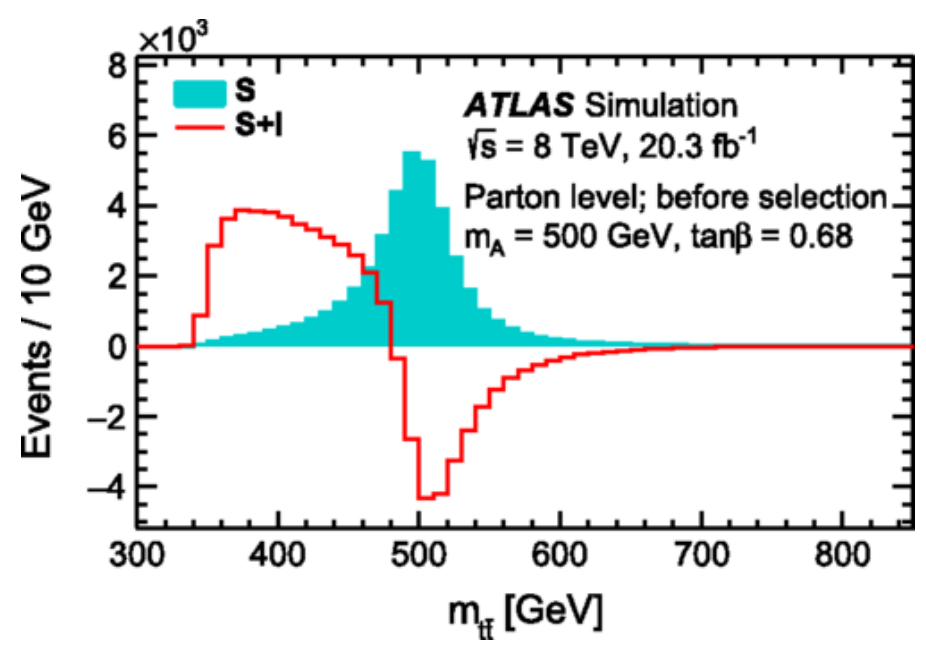

Figure 5: Invariant mass of the $t \bar{t}$ pair from the decay of a BSM Higgs with mass $500 \mathrm{GeV}$, before the emission of final state radiation and parton showering. The filled blue area shows the distribution from a pure signal resonance and the red line is from the signal plus interference [10].

The analysis targets the $t \bar{t} \rightarrow W b W b$ decay channel, with one hadronic and one leptonic $W$ decay. Therefore, events are selected by a single lepton trigger and require an isolated lepton with transverse momentum above $25 \mathrm{GeV}$. There are 4 hadronic jets in the final state and at least one of those must be a $b$-jet. It is split into three channels based on the whether the $b$-tag is with the hadronic $W$ decay, leptonic $W$ decay, or with both $W$.

The main background is the Standard Model $t \bar{t}$, which is estimated using simulation. First, a MaDGraph5_aMC@NLO [11] sample is generated for the signal plus interference contribution only. the Standard Model $t \bar{t}$ contribution is removed from this, and instead replaced by a more reliable NLO simulation from Powheg-Box + Pythia6 [12, 13]. Additional backgrounds from $W+$ jets and multijet processes are estimated using data-driven methods.

The expected standard model background and observed data is in good agreement. A profile likelihood fit to the reconstructed mass of the $t \bar{t}$ is conducted for all signal regions simultaneously. Then the exclusion limits for each model are derived from that fit. The post-fit distribution is shown in Fig. 6. Limits are set in the $\tan \beta$ against $m_{A}$ plane for some particular models, as shown in Fig. 7.

Another probe of BSM Higgs coupling to top quarks is via the associated production, $(b \bar{b} / t \bar{t})+$ $A / H \rightarrow t \bar{t}$. Although there is no dedicated ATLAS search for BSM Higgs bosons in this channel, results from a search for $t \bar{t}$ final plus heavy flavour jets was reinterpreted for 2 HDMs. The advantage of this channel is that there is negligible interference between signal and backgrounds. However, it has complicated final states. The reinterpretation in terms of BSM Higgs was presented in Ref. [14]. 


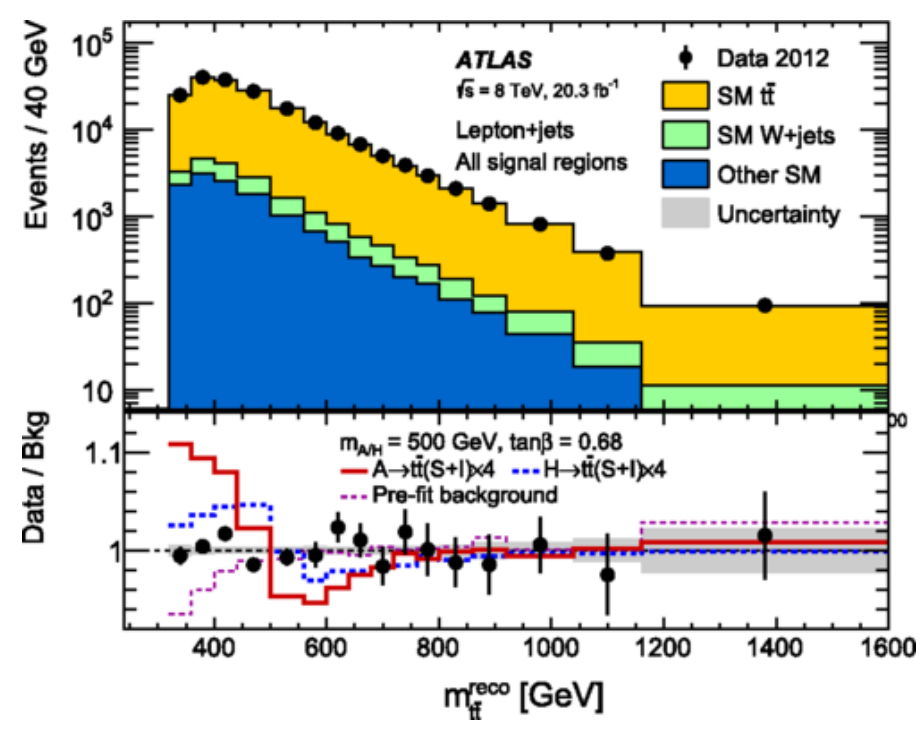

Figure 6: Distribution of the reconstructed top pair mass in the BSM Higgs to $t \bar{t}$ analysis. The data and background are shown after the profile likelihood fit with the background-only hypothesis. All of the signal regions are combined in the fit. In bottom panel for the ratio of data to background, two signal models are shown: a pseudoscalar (A) and scalar (H) BSM Higgs boson, with $m=$ $500 \mathrm{GeV}$ and $\tan \beta=0.68$. The signal has been scaled by a factor 4 for illustration [10].

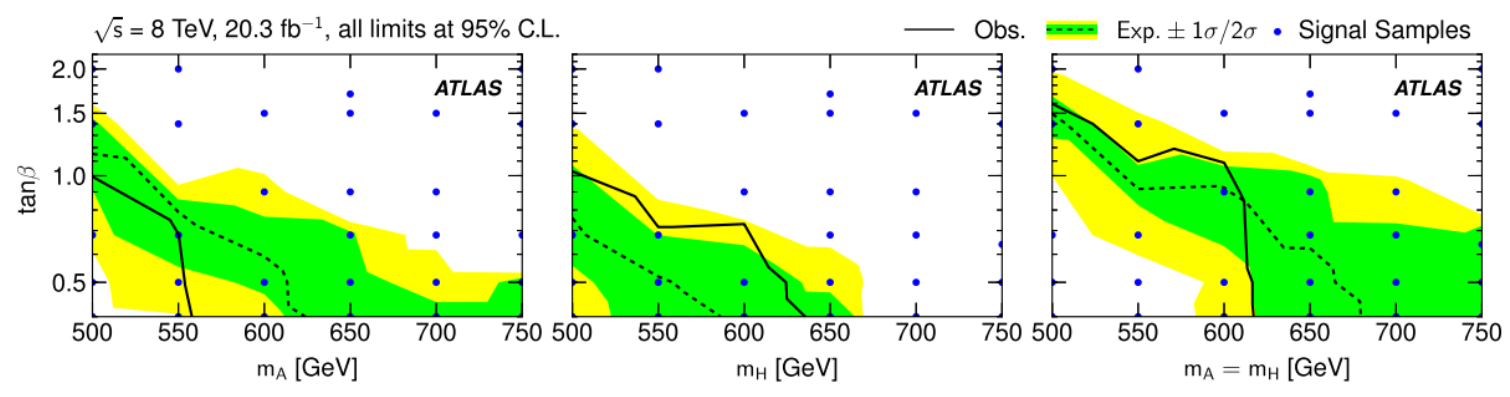

Figure 7: Observed and expected 95\% exclusion limits for type-II 2HDM from the $H / A \rightarrow t \bar{t}$ search. The left panel considers only a pseudoscalar Higgs, the middle only a scalar Higgs, and the right panel the degenerate scenario where the scalar and pseudoscalar have equal mass [10].

\section{Conclusions and outlook}

Searches for heavy neutral BSM Higgs bosons via fermionic decays at ATLAS have significantly improved limits over previous results. In particular, the complementarity between $\tau$ and top decay channels means limits can be set for both high and low $\tan \beta$ in the MSSM. So far results have been from LHC Run 1 data $(\sqrt{s}=8 \mathrm{TeV})$, or early Run 2 data $(\sqrt{s}=13 \mathrm{TeV})$, with a maximum of $36.1 \mathrm{fb}^{-1}$ data collected. Now that Run 2 of the LHC has been completed (December 2018), the full dataset at $\sqrt{s}=13 \mathrm{TeV}$ will be available. This will give approximately $150 \mathrm{fb}^{-1}$ of proton-proton collisions in total, providing a significant increase over the current results. With this there is further opportunity for discovery, or to exclude even larger regions of parameter space of 2HDMs. 


\section{References}

[1] ATLAS Collaboration, Observation of a new particle in the search for the Standard Model Higgs boson with the ATLAS detector at the LHC, Phys. Lett. B, 716 (2012) 1 [hep-ex/1207. 7214 ].

[2] CMS Collaboration, Observation of a new boson at a mass of $125 \mathrm{GeV}$ with the CMS experiment at the LHC, Phys. Lett. B, 716 (2012) 1 [hep-ex/1207 . 7235].

[3] G. C. Branco et al., Theory and phenomenology of two-Higgs-doublet models, Phys. Rept., 516 (2012) 1 [hep-ph/1106.0034].

[4] A. Djouadi, The Anatomy of electro-weak symmetry breaking. II. The Higgs bosons in the minimal supersymmetric model, Phys. Rept. 459 (2008) 1, [hep-ph / 0503173 ].

[5] A. Djouadi et al., The post-Higgs MSSM scenario: Habemus MSSM?, Eur. Phys. J. C 73 (2013) 2650, [hep-ph/1307.5205].

[6] E. Bagnaschi et al., Benchmark scenarios for low $\tan \beta$ in the MSSM, LHCHXSWG-2015-002, (2015), http://cdsweb.cern.ch/record/2039911

[7] M. Carena, S. Heinemeyer, O. Stål, C. Wagner and G. Weiglein, MSSM Higgs boson searches at the LHC: benchmark scenarios after the discovery of a Higgs-like particle, Eur. Phys. J. C 73 (2013) 2552, [hep-ph/1302.7033].

[8] ATLAS collaboration, Search for additional heavy neutral Higgs and gauge bosons in the ditau final state produced in $36 \mathrm{fb}^{-1}$ of pp collisions at sqrts $=13 \mathrm{TeV}$ with the ATLAS detector, J. High Energy Phys., (2018) 55. [hep-ex/1709.07242].

[9] ATLAS Collaboration, Reconstruction of hadronic decay products of tau leptons with the ATLAS experiment, Eur. Phys. J. C, 76 (2016) 295 [hep-ex/1512 . 05955].

[10] ATLAS Collaboration, Search for Heavy Higgs Bosons A/H Decaying to a Top Quark Pair in pp Collisions at $\sqrt{s}=8$ TeV with the ATLAS Detector, Phys. Rev. Lett. 119, (2017) 191803 [hep-ex/1707.06025].

[11] J. Alwall, R. Frederix, S. Frixione, V. Hirschi, F. Maltoni, O. Mattelaer, H.-S. Shao, T. Stelzer, P. Torrielli, and M. Zaro, The automated computation of tree-level and next-to leading order differential cross sections, and their matching to parton shower simulations, J. High Energy Phys. 07 (2014) 079 [hep-ph/1405.0301].

[12] S. Frixione, P. Nason, and G. Ridolfi, A positive-weight next-to-leading-order Monte Carlo for heavy flavour hadroproduction, J. High Energy Phys. 09 (2007) 126 [hep-ph / 0707 . 3088].

[13] T. Sjöstrand, S. Mrenna, and P. Z. Skands, PYTHIA 6.4 Physics and Manual, J. High Energy Phys. 05 (2006) 026 [hep-ph/ 0603175$].$

[14] ATLAS Collaboration, Search for new phenomena in $t \bar{t}$ final states with additional heavy-flavour jets in pp collisions at $\sqrt{s}=13 \mathrm{TeV}$ with the ATLAS detector ATLAS-CONF-2016-104 (2016). 